\title{
THE INFLUENCE OF NANO-SILVER ON FORMATION OF MICROBIAL BIOFILMS IN CASES OF TRAUMATIC LESION OF THE AUXILIARY APPARATUS OF THE EYE
}

\author{
Oksana Petrenko \\ visionpetrenko@gmail.com \\ Maryna Dranko ${ }^{1}$ \\ Department of eye microsurgery ${ }^{2}$ \\ drankoma@ukr.net \\ Victoriia Holubnycha \\ Public health department ${ }^{2}$ \\ golubnichiy@ukr.net \\ Larysa Hrytsai \\ Department of eye microsurgery ${ }^{2}$ \\ hrytsay_larysa62@mail.com \\ ${ }^{1}$ Department of Ophthalmology \\ Shupyk National Medical Academy of Postgraduate Education \\ 9 Dorohozhytsra str., Kyiv, Ukraine, 04112 \\ ${ }^{2}$ Sumy region clinical hospital \\ 48 Troitska str., Sumy, Ukraine, 40022
}

\begin{abstract}
Pyoinflammatory complications remain an acute problem in the post-operative period of traumatic lesions of the auxiliary apparatus of the eye (AAE). Silver both in the ionic form and in composition of chemical compounds is highly toxic for microorganisms, and as a result, it shows bactericidal effect to many bacterial strains, including gram-negative microorganisms. The peculiarity of AgNPs is efficiency of influence on the wide array of microorganisms, significant anti-biofilm effect and absence of resistance reaction.

The aim of the research. To study the influence of the colloidal nano silver on formation of biofilms by microorganisms discharged from the wounds of patients with traumatic lesions of the auxiliary apparatus of the eye.

Materials and methods. During 2018-2019, we examined 60 patients with traumatic lesions of the auxiliary apparatus of the eye. For evaluation of the influence of colloid nano silver solution on the processes of formation of the biofilm, we selected microorganisms which were cultured most frequently (Staphylococcus aureus, Acinetobacter spp., Klebsiella ozenae) from the patients.

Results. The obtained data suggest that colloid nano silver inhibits efficiently formation of biofilms at the early stages (initiation, the 0 day of incubation) of their formation by all the three microorganisms, and the degree of inhibition of the biofilm formation did not depend on the silver concentration.

The effect of colloid silver in the concentrations used by us at later stages of biofilm formation (the $3^{\text {rd }}$ and the $7^{\text {th }}$ day) with respect to K. ozenae is less efficient - the growth of cell biomass was observed ( $p \leq 0.05)$, and it did not depend on the silver concentration. At the same time, the effect of the colloid nano silver on S. aureus and Acinetobacter spp. on the $3^{\text {rd }}$ and the $7^{\text {th }}$ days was more efficient than at the early stage $(\mathrm{p} \leq 0.05)$.

Conclusions. Nanoparticles of colloid silver are an efficient means to combat biofilms, as well as to prevent their formation.

Keywords: colloidal nano silver, biofilms, minimum inhibitory concentration (MIC), trauma of the auxiliary apparatus of the eye, infection.
\end{abstract}

DOI: $10.21303 / 2504-5679.2020 .001329$

\section{Introduction}

Pyoinflammatory complications remain an acute problem in the post-operative period of traumatic lesions of the auxiliary apparatus of the eye (AAE), since they cause delayed wound healing and provoke formation of adverse vicious cicatrices $[1,2]$. For prevention of pyoinflammatory 
complications and chronization of processes of wound healing it is important to carry out monitoring of the specious composition and sensitivity of microorganisms which are discharged from the traumatic lesions of the patients to the most common antibiotics $[3,4]$.

At the present stage of the development of medicine and pharmacology, a quite acute problem is the development of chemotherapeutic agents, which are an alternative to antibiotics, since in the clinical practice in the whole world a steep increase of the number of resistant microorganism strains [5,6]. Antibacterial properties of silver are known from a long time. Silver both in the ionic form and in composition of chemical compounds is highly toxic for microorganisms, and as a result, it shows bactericidal effect to many bacterial strains, including gram-negative microorganisms [7,8]. Antimicrobial efficiency of silver depends on the size of the particles, antibacterial activity of the nanoparticles surpasses activity of the common silver solutions [9, 10]. It is proved that nanoparticles of silver (AgNPs) affect penetrability of bacterial membranes and adhesion of bacteria to the cell membrane $[11,12]$. AgNPs also affects membrane and intracellular proteins of bacteria, remnants of phosphoric acid and DNA, distorts cell division, causing their death $[13,14]$. So, the peculiarity of AgNPs is efficiency of influence on the wide array of microorganisms, significant anti-biofilm effect and absence of resistance reaction $[15,16]$.

Methods of treatment of wounds with biofilms must include wound cleansing with application of antiseptic and antimicrobial medicines for prevention of wound re-infection and inhibition of the repeated formation of biofilms [17, 18].

The aim of the research. To investigate the influence of the colloidal nano silver on formation of biofilms by microorganisms discharged from the wounds of patients with traumatic lesions of the auxiliary apparatus of the eye.

\section{Materials and methods}

During 2018-2019, we examined 60 patients with traumatic lesions of the auxiliary apparatus of the eye, who sought help at the emergency room at the Eye Microsurgery Department of the Municipal Non-commercial Enterprise of Sumy Regional Council Sumy Regional Clinical Hospital. Examination and treatment of the patients were carried out according to the standards of provision of the medical assistance. The procedure of examination of those persons met the regulations of the ethics committee. At the preliminary stage bacteriologic examination of smears from the wounds was carried out, with identification of the specious composition and population level of microorganisms based on the bacteriologic laboratory of the Centre of the Collective Use of Scientific Equipment of the Medical Institute of Sumy State University. For evaluation of the influence of colloid nano silver solution on the processes of formation of the biofilm, we selected microorganisms which were cultured from the patients most frequently (Staphylococcus aureus, Acinetobacter spp., Klebsiella ozenae).

This clinical research is met Ukrainian legislation, modern ethical standards and principles of scientific clinical research and was approved at the meeting of the Commission on Ethics of the Shupyk National Medical Academy of Postgraduate Education (protocol No. 1 of January 9, 2020). All patients provided informed written consent to participate in the study.

Two groups were formed: the control one (microorganisms without influence of the colloid nano silver solution) and the main one (microorganisms were exposed to the colloid nano silver solution). The colloid nano silver solution (Nanomaterialy i Nanotekhnologii (Nanomaterials and Nanotechnologies) LLC, Ukraine, registration number 05.03.02-04/66400 as of 16.09.2010) was added to the microorganisms for $0,1,3$ and 7 days of incubation in the amount equal to 2.5, 5, 10 minimum inhibitory concentrations for every specious of microorganisms.

Evaluation of the influence of silver nanoparticles on the formation of biofilms was carried out by the definition of the volume of the formed biofilm, with gentian violet staining [19, 20]. The optical density of microbial biomass was defined by the Thermo Scientific Multiscan FC microplate photometer ESW 1.01.16 (wavelength $595 \mathrm{~nm}$ ). The coefficient of the microbial biomass reduction was calculated as a proportion of the optical density of the tested sample to the optical density of control in the percentage equivalent. 
The results of the performed investigations underwent statistical processing. The GraphPadQuikCalcs program with the definition of t-Student criterion was used for carrying out the calculation.

\section{Results}

Sixty patients with traumatic lesions of the auxiliary apparatus of the eye were examined. Seventy-five strains of microorganisms were isolated and identified from them. Considering the frequency of microorganisms isolated from the patients with lesions of the AAE, we selected Staphylococcus aureus, Acinetobacter spp., Klebsiella ozenae for study the influence of colloid nanosilver on the formation of biofilm.

We tested antimicrobial and antibiofilm properties at different concentrations of the colloidal nanosilver. We found the MIC for planktonic cultures of clinical isolates of S. aureus, Acinetobacter spp., K. ozenae was equal to $12.5 \mathrm{~m} \mu / \mathrm{ml}$.

The adhesion of bacterial cells to the surface is considered the first step for biofilm formation. Consequently, in the beginning, we examined the influence of silver nanoparticles on bacteria adhesion. The obtained data (Fig. 1) suggest that colloid nanosilver inhibits the formation of biofilms at the beginning stage efficiently. The degree of the biofilm formation inhibition for Klebsiella and Staphylococcus was dependent on the silver concentration and was not dependent on the quantity of the silver for Acinetobacter spp.

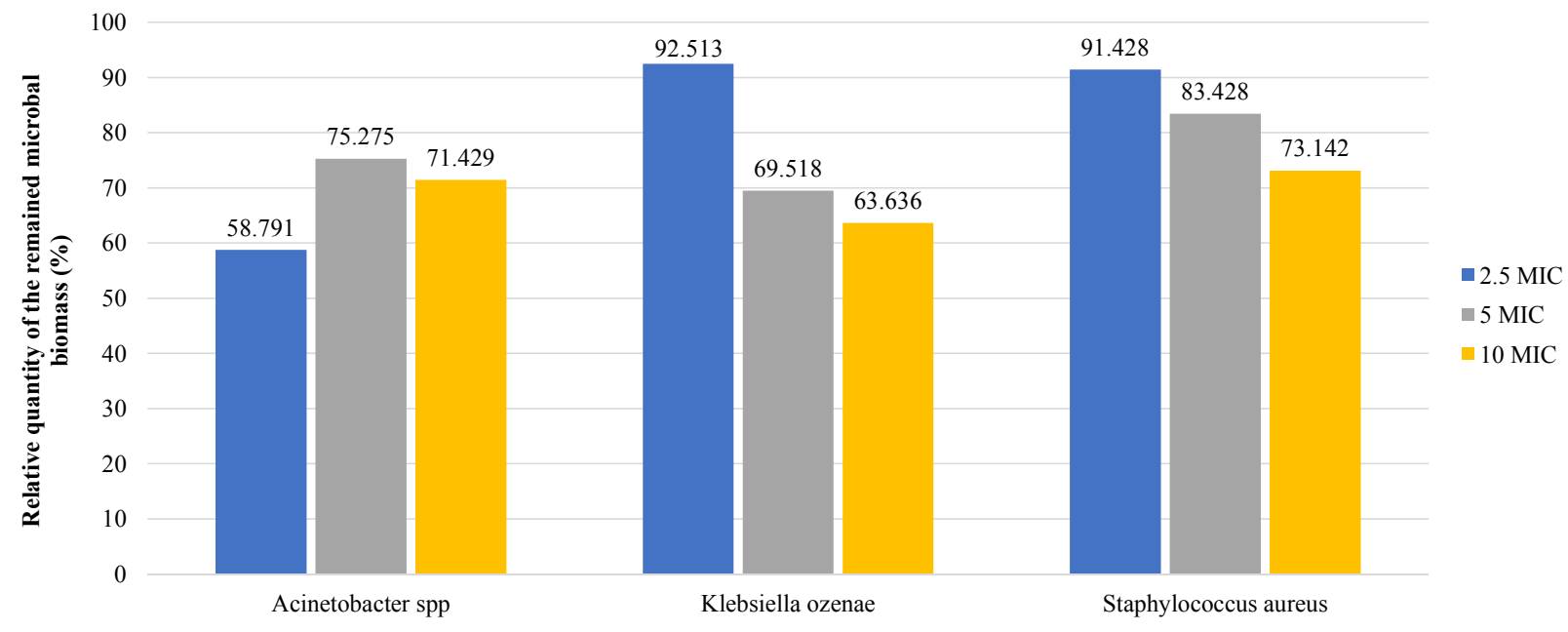

Fig. 1. Influence of nanosilver on the immature biofilms (initiation)

As it is shown in Fig. 2-4, we have revealed the intense activity in colloidal nanosilver against mature biofilms formed by all types of tested microorganisms. The efficiency of silver increased directly with the growth of the silver amount in the culture medium. Moreover, the silver acts differently on biofilms with various stages of maturation. It was especially noticeable on the one-day films. The biofilms formed by Acinetobacter spp.was the most susceptible to the influence of nanosilver. The relative quantity of the remaining bacteria cells in the biofilm was the least after treatment among all species. S. aureus formed the most stable composition of the biofilm since the remained biofilm mass varied from 71.9 to 55.8 (44.3\%) after silver processing.

The effect of colloid silver at later stages of biofilm formation (the $3^{\text {rd }}$ and the $7^{\text {th }}$ day) against K. ozenae and Acinetobacter spp. is less efficient (Fig. 3, 4) than at an early stage. The volume of the cell biomass after silver action was more significant $(\mathrm{p} \leq 0.05)$ comparing to the 1-day biofilm. There was not also a correlation between the silver concentration and remained biomass. At the same time, the effect of the colloid nanosilver on S.aureus biofilm on the $3^{\text {rd }}$ and the $7^{\text {th }}$ days was more efficient than at the early stage $(p \leq 0.05)$. 


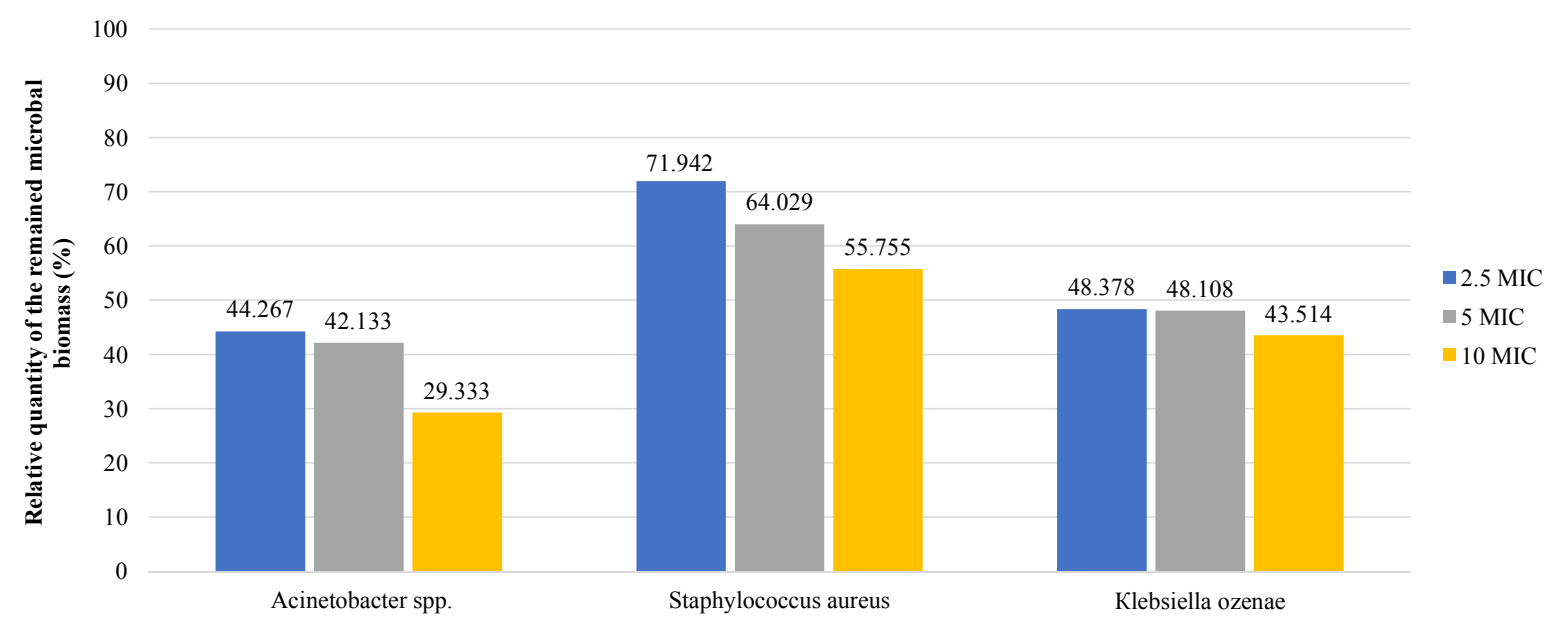

Fig. 2. Influence of nanosilver on the mature biofilms (1day)

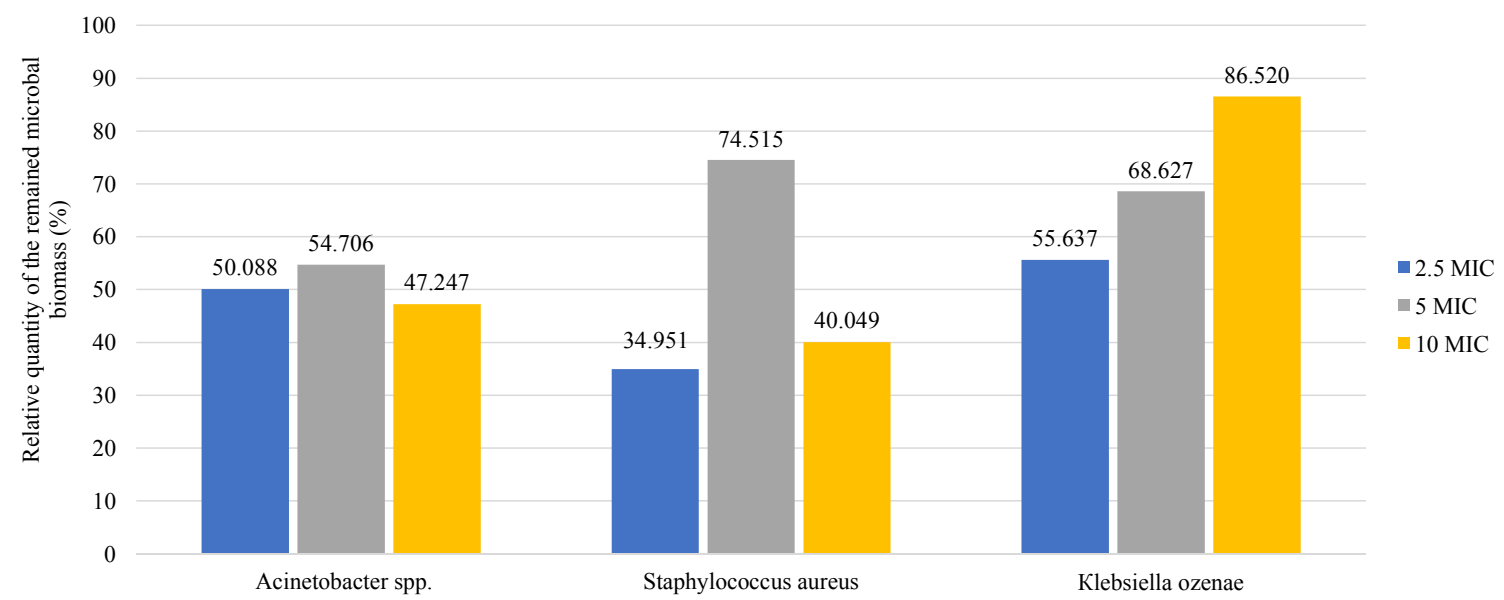

Fig. 3. Influence of nanosilver on the mature biofilm (3 days)

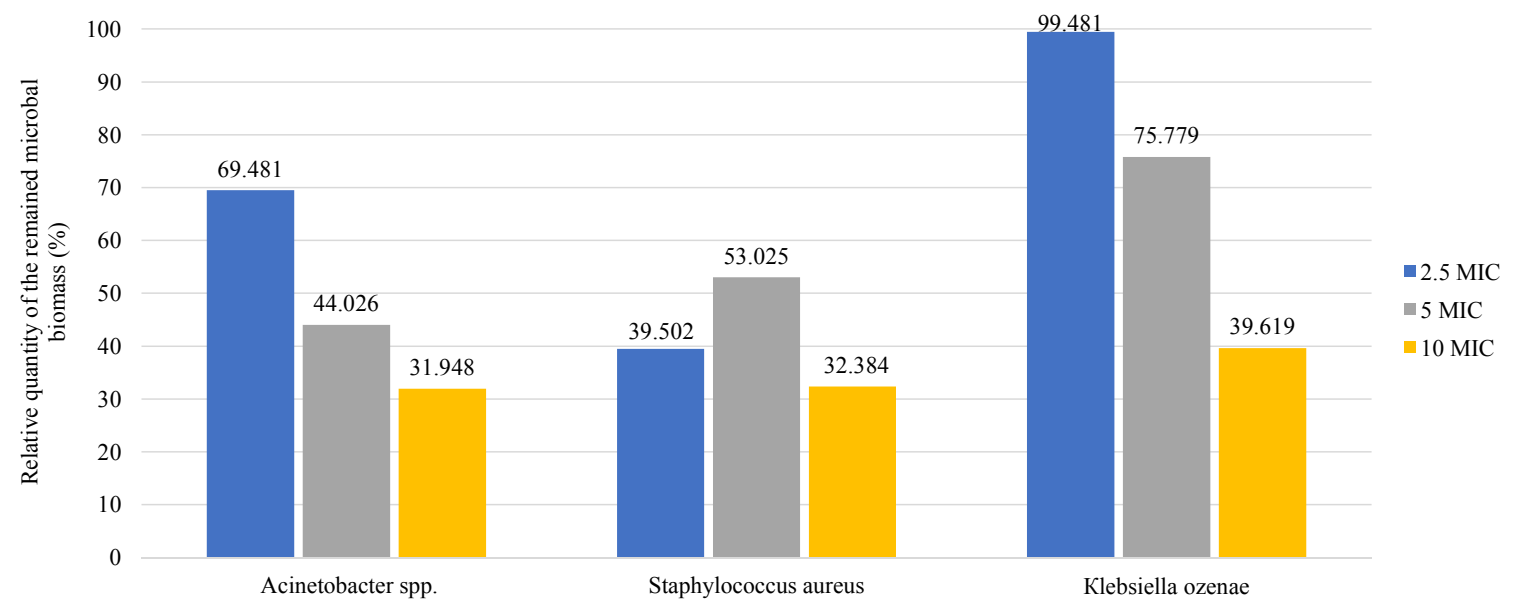

Fig. 4. Influence of nanosilver on the mature biofilm (7 days)

\section{Discussion}

Attention to wound infection is critical for quick repair with no side effects. It gains exceptional value due to the rapid growth of antibiotic resistance and insufficient needs to search for new medicine with antimicrobial activity. Nanomaterials have become a promising alternative to 
conventional antibiotics. It was shown in several studies that AgNPs possesses broad-spectrum antibacterial activities [7, 21]. The increasing number of studies have demonstrated the presence of biofilm in wounds of varied etiology [22]. The wound is inevitably created under aseptic surgical conditions. The prevention of bacteria entering the wound is based on the interruption of microbial attachment to the tissue on an initial step [22]. In this study, we report the capabilities of colloidal nanosilver to prevent the formation of biofilms by Acinetobacter spp., Klebsiella ozenae, and S. aureus. According to other scientist data, the minimum biofilm eradication concentration for antiseptics exceed MIC for planktonic bacteria more than 10-1000 times [3, 4]. We revealed that colloidal nanosilver effectively destroys the biofilms formed by microorganisms isolated from patients with traumatic lesions of the auxiliary apparatus of the eye even at a concentration equal to $2.5 \mathrm{MIC}$. The effect of the nanosilver varied in different terms and was the highest on 1-day films. Probably the reason for such variation was the difference in biofilm composition. The immature biofilm consists mostly of cells that are more sensitive to the silver influence than exopolysaccharide matrix. It is known that ionic silver may be inactivated by extracellular polymeric substances (EPS) and other organic matter within the biofilm [23].

Study limitations. Our research is limited by studying the effect of nanosilver on the formation of biofilms by microorganisms isolated from traumatic wounds of the auxiliary apparatus of the eye.

Prospects for further research. The further investigation of other nanometals effect on the biofilms formed by microorganisms isolated from patients with lesions of the eyes will provide us new knowledge.

\section{Conclusions}

1. As a result of the clinical-experimental research, we received a positive effect of colloidal nanosilver at the concentration $31.5-125.0 \mu \mathrm{g} / \mathrm{ml}$ on the biofilms formed by $S$. aureus, Acinetobacter spp., Klebsiella ozenae.

2. Nanosilver inhibits the formation of biofilms at the beginning stage efficiently. The degree of the biofilm formation inhibition for Klebsiella and Staphylococcus was dependent on the silver concentration and was not dependent on the quantity of the silver for Acinetobacter spp..

3. The intense activity in colloidal nanosilver against mature biofilms formed by all types of tested microorganisms. The efficiency of silver increased directly with the growth of the silver amount in the culture medium.

4. The silver acts differently on biofilms with various stages of maturation.

Colloidal nanosilver could be used for the prevention of complicated wound infection in patients with traumatic lesions of the auxiliary apparatus of the eye. Use of colloidal silver could reduce the risk of post-traumatic deformations of the periorbital region, decrease the duration and cost of treatment as well as period of rehabilitation.

\section{Acknowledgments}

This work was supported by the Government Program "State order for scientific-technical (experimental) development and scientific and technical production”. Project 0118U003577 Effectiveness of chitosan-nanometals antimicrobial action against clinical multiresistant strains.

\section{Conflict of interest}

The authors declare that they have no conflicts of interest.

\section{References}

[1] Petrenko, O. V. (2015). Posttraumatic defects of the eye auxiliary apparatus (anatomic-functional and esthetic disorders, clinical picture, treatment, efficiency prognosis). The Archive of Ophthalmology of Ukraine, 2 (3), 38-43.

[2] Petrenko, O. M., Bezrodnyi, B. G., Bondarchuk, O. L. (2016). Formation of biofilms by soft tissue phlegmon pathogens. Surgery of Ukraine, 1, 85-89.

[3] Rakhmatulina, M. R., Nechaeva, I. A. (2015). Biofilms of microorganisms and their role in forming resistance to antibacterial medicines. Newsletter of Dermatology and Venereology, 2, 58-62. 
[4] Svizhak, V. K., Danchuk, A. H., Deineka, S. Ye. (2015). The local monitoring of antibiotic susceptibility of the main causative agents of pyoinflammatory infections. Part 1 . Taxonomic composition of microbiota that forms inflammatory process. Clinical and Experimental Pathology, 3, 113-116.

[5] Vickery, K. (2019). Special Issue: Microbial Biofilms in Healthcare: Formation, Prevention and Treatment. Materials, 12 (12), 2001. doi: http://doi.org/10.3390/ma12122001

[6] Svizhak, V. K., Danchuk, A. H., Deineka, S. Ye. (2015). The local monitoring of antibiotic susceptibility of the main causative agents of pyoinflammatory infections. Part 1 . Taxonomic composition of microbiota that forms inflammatory process. Clinical and Experimental Pathology, 4, 143-150.

[7] Gladkikh, P. G. (2015). Effect of nanoparticles of silver in regards to biofilms of microorganisms (review of literature). Newsletter of New Medical Technologies, 1 (3-4).

[8] Synetar, E. A. (2015). The influence of silver nanoparticles of the formation of biofilm bacteria Enterococcus faecalis. Newsletter of Issues of Biology and Medicine, 4 (1 (124)), 201-205.

[9] Sukhina, M. A., Shelygin, Y. A., Piyadina, A. Y., Feldman, N. B., Ananyan, M. A., Lutsenko, S. V., Frolov, S. A. (2019). The inhibitory and destructive action of the silver nanoparticle preparation on biofilms formed by clinically relevant microorganisms. Koloproktologia, 18 (3 (69)), 56-70. doi: http://doi.org/10.33878/2073-7556-2019-18-3-56-70

[10] Dong, Y., Zhu, H., Shen, Y., Zhang, W., Zhang, L. (2019). Antibacterial activity of silver nanoparticles of different particle size against Vibrio Natriegens. PLOS ONE, 14 (9), e0222322. doi: http://doi.org/10.1371/journal.pone.0222322

[11] Tudose, M., Culita, D. C., Munteanu, C., Pandele, J., Hristea, E., Ionita, P. et. al. (2015). Antibacterial Activity Evaluation of Silver Nanoparticles Entrapped in Silica Matrix Functionalized with Antibiotics. Journal of Inorganic and Organometallic Polymers and Materials, 25 (4), 869-878. doi: http://doi.org/10.1007/s10904-015-0176-7

[12] Akter, M., Sikder, M. T., Rahman, M. M., Ullah, A. K. M. A., Hossain, K. F. B., Banik, S. et. al. (2018). A systematic review on silver nanoparticles-induced cytotoxicity: Physicochemical properties and perspectives. Journal of Advanced Research, 9, 1-16. doi: http://doi.org/10.1016/j.jare.2017.10.008

[13] Derkach, S. A., Voronkina, I. A., Habysheva, L. S., Krylov, I. A., Kutsay, N. M. (2016). Antibiotics impact on formation of biofilms mssa and mrsa strains of staphylococci. Infectious Diseases, 4, 46-51. doi: http://doi.org/10.11603/1681-2727.2016.4.7212

[14] Vo, D.-T., Sabrina, S., Lee, C.-K. (2017). Silver deposited carboxymethyl chitosan-grafted magnetic nanoparticles as dual action deliverable antimicrobial materials. Materials Science and Engineering: C, 73, 544-551. doi: http://doi.org/10.1016/j. msec.2016.12.066

[15] Pinto, R. M., Lopes-de-Campos, D., Martins, M. C. L., Van Dijck, P., Nunes, C., Reis, S. (2019). Impact of nanosystems in Staphylococcus aureus biofilms treatment. FEMS Microbiology Reviews, 43 (6), 622-641. doi: http://doi.org/10.1093/femsre/ fuz021

[16] Bhattacharya, M., Wozniak, D. J., Stoodley, P., Hall-Stoodley, L. (2015). Prevention and treatment ofStaphylococcus aureusbiofilms. Expert Review of Anti-Infective Therapy, 13 (12), 1499-1516. doi: http://doi.org/10.1586/14787210.2015.1100533

[17] Arciola, C. R., Campoccia, D., Montanaro, L. (2018). Implant infections: adhesion, biofilm formation and immune evasion. Nature Reviews Microbiology, 16 (7), 397-409. doi: http://doi.org/10.1038/s41579-018-0019-y

[18] Roberts, A. E. L., Kragh, K. N., Bjarnsholt, T., Diggle, S. P. (2015). The Limitations of In Vitro Experimentation in Understanding Biofilms and Chronic Infection. Journal of Molecular Biology, 427 (23), 3646-3661. doi: http://doi.org/10.1016/ j.jmb.2015.09.002

[19] Okulich, V. K., Kabanova, A. A., Plotnikov, F. V. (2017). Microbial biofilms in clinical microbiology and antibacterial therapy. Vitebsk: Vitebsk State Medical University, 300.

[20] Simonova, I. R., Golovin, S. N., Verkina, L. M., Berezniak, Ye. A., Titova, S. V. (2017). Methods of cultivation and study of bacterial biofilms. Izvestiya VUZov. North Caucasian District, 1, 73-79.

[21] Lara, H. H., Romero-Urbina, D. G., Pierce, C., Lopez-Ribot, J. L., Arellano-Jiménez, M. J., Jose-Yacaman, M. (2015). Effect of silver nanoparticles on Candida albicans biofilms: an ultrastructural study. Journal of Nanobiotechnology, 13 (1). doi: http:// doi.org/10.1186/s12951-015-0147-8

[22] Percival, S. L., McCarty, S. M., Lipsky, B. (2015). Biofilms and Wounds: An Overview of the Evidence. Advances in Wound Care, 4 (7), 373-381. doi: http://doi.org/10.1089/wound.2014.0557

[23] Gloag, E. S., Fabbri, S., Wozniak, D. J., Stoodley, P. (2020). Biofilm mechanics: Implications in infection and survival. Biofilm, 2, 100017. doi: http://doi.org/10.1016/j.bioflm.2019.100017 